\title{
POSTER PRESENTATION - 1
}

CUTTING EDGE TECHNOLOGIES IN CLINICAL BIOCHEMISTRY 
P1.1

\title{
Clinical Utility of Vitros dTIBC in Assessment of Iron Deficiency
}

Vidya Kakade, Nita Munshi

Ruby Hall Clinic, Pune

To correlate the results of serum iron \& direct TIBC and \% Saturation with clinical symptoms and history of patients $(\mathrm{N}=32)$ admitted in a leading hospital at Pune.

Serum specimens were obtained from the patients $(N=32)$ suspected of Iron deficiency. Serum Iron tests were measured using VITROS MicroSlide technology and the direct TIBC (dTIBC) was measured by the VITROS Micro Tip technology, both on fully automated random access VITROS 5, 1 FS Fusion analyzer. Percentage Saturation was automatically calculated by the instrument using the formula : $(\mathrm{Fe} / \mathrm{TIBC})$ * 100.

The results were compared with Haemoglobin, $\mathrm{MCV}, \mathrm{MCH}$ along with clinical symptoms and history of patients.

Out of 32 patients, 1 patient had increased Serum Iron level, 19 patients had reduced Serum Iron levels and rest 12 patients were with normal level of Serum Iron. 19 patients with reduced serum iron levels were further investigated.

Out of 19 patients with low serum iron levels, 5 with reduced levels for all the parameters (FE, dTIBC, $\%$ Saturation) indicates infection / inflammation; and the other 14 patients with low serum iron level and highly reduced \% Saturation level and normal dTIBC value indicates Iron deficiency.

dTIBC test helps for the differential diagnosis of Iron deficiency and other infection / inflammation. Shorter turn around time, as low as 14 mins for dTIBC of VITROS MicroTip assay on VITROS 5, 1 FS helped the clinician for necessary action with the help of the results.

P1.2

\section{Humanization of Murine Anti-HBs Antibody by Genetic Manipulation of the Framework Residues with the Aid of Molecular Modeling}

\author{
Ashutosh Tiwari ${ }^{1}$, Navin Khanna ${ }^{2}$, Subrata K Acharya ${ }^{3}$, Subrata Sinha ${ }^{1}$ \\ 1 Department of Biochemistry, All India Institute of Medical Sciences, New Delhi \\ 2 Recombinant Gene Product Lab, ICGEB, New Delhi \\ ${ }^{3}$ Department of Gastroenterology, All India Institute of Medical Sciences, New Delhi
}

Antibodies against Hepatitis B virus (HBV) are used either as stand-alone therapy or as adjunct for managing chronic viral infection. Our group developed a mouse monoclonal antibody (5S) against the ' $a$ ' epitope of the Hepatitis B surface antigen $(\mathrm{HBsAg})$, which is the major domain for both diagnosis and 
Indian Journal of Clinical Biochemistry, December 2007, Vol. 22 (Supl.)

immunoprophylaxis of HBV. However, this mouse antibody cannot be used for therapeutic purposes, as it may elicit human anti-mouse immune responses. Humanization of $5 \mathrm{~S}$ antibody can make it more suitable for possible clinical use by reducing the immunogenicity of this antibody. In this study we report the humanization of scFv form of high affinity antibody against Hepatitis B surface antigen.

We constructed a molecular model of scFv by web based modeling software. We selected non human like (immunogenic) residue by analyzing the frequency of particular residues in the human database. Among the selected residues, only those were mutated which were not showing any structural discrepancy with in the context of molecular model of 5S-scFv. Selected non human like residues were subsequently mutated to human residues by site-directed mutagenesis. After initial screening using the phage display system, the humanized scFv was expressed in soluble form in $E$. coli.

We characterized the humanized scFv using several in-vitro techniques and it was observed that the humanized scFv retained the high binding affinity (nano molar range) to HBsAg and specificity of the original mouse monoclonal. The humanized scFv also maintained antigen binding in the presence of various destabilizing agents like $3 \mathrm{M} \mathrm{NaCl}, 30 \%$ DMSO and $8 \mathrm{M}$ urea.

We have generated a humanized anti-HBs scFv antibody without any loss in binding affinity and epitope specificity. Therefore, further manipulation of this humanized scFv can lead us towards the whole humanized $\lg G$ derivatives, which can be safely utilized for passive therapy in case of HBV infection.

\title{
P1.3
}

\section{Integration of Bacteriophage Sequence in the Human Genome}

\author{
Manish Kumar Sharma, Sachin Puri, Geeta Jotwani, Jyoti Gupta, Ejaz Hussain, Chitra \\ Sarkar, A.K. Mahapatra, Subrata Sinha \\ Department of Biochemistry, All India Institute Medical of Sciences, New Delhi
}

Identification and characterization of genetic alterations are very important in understanding and deciphering the exact molecular events involved in tumorigenesis. Study of genetic alterations will allow a better interpretation of the interplay between genes that are involved in the induction of meningiomas. Previous work in our laboratory suggests the importance of using RAPD in analyzing an overall instability in the genome under study. The results indicated four different genetic loci were associated with genome of meningiomas, designated as meng- 1 , meng- 2 , meng-3 and meng- 4 . In order to identify the genetic alterations, it is decided to concentrate on meng-1 because of homology with the bacteriophage and the absence till date of any information regarding the integration of the bacteriophage sequence in the human genome and their association with tumorigenesis.

Using different sets of overlapping primers based on bacteriophage HKO22 sequences flanking the meng1 sequence we have been able to PCR amplify different stretches of phage corresponding to the tumor and normal (blood) genome. Genome walking was also performed towards the $5^{\prime}$ end of bacteriophage sequence to find out the junction in the human genome. 
Indian Journal of Clinical Biochemistry, December 2007, Vol. 22 (Supl.)

We have been able to amplify a continuous stretch corresponding to nucleotide 69 to nucleotide 5736 of the bacteriophage (meng-1 was homologous from nucleotide 1880 to nucleotide 31 10). From nucleotide 115, genome walking was done in the direction of $5^{\prime}$ end. At the $5^{\prime}$ end the next nucleotide was nucleotide 40,751 after the nucleotide 1 and then continued till nucleotide 40,132.

The meng-1 sequence has a very high degree of homology with the bacteriophage HKO22, indicating the integration of phage sequences in the human genome causing meningioma. At the point of 5 'end of sequence, the continuation of 40,751 to 40,132 indicates recircularization of bacteriophage genome before integration in human genome.

$\mathrm{P} 1.4$

\title{
Prevalance of Hemochromatosis in North Indians
}

\author{
Shweta Saxena', Rahul Sharma ${ }^{3}$, Sarita Agarwal ${ }^{3}$, S.P.Saxena', Ajai Kumar ${ }^{2}$ \\ ' B.R.D. Medical College, Gorakhpur; ${ }^{2}$ G.S.V.M. Medical College, Kanpur; ${ }^{3}$ Sanjay Gandhi PGIMS, \\ Lucknow.
}

Hemochromatosis is an Autosomal recessive \& most commonly single gene inherited disorder among caucasions with prevalance of 5/1000 and a carrier frequency of 1 in 10. Mainly two point mutations have been described which include C282Y \& H63D. Knowledge of the prevalence of the genes influencing iron overload would ensure preventive treatment of iron overload related disorders. Present study has been carried out due to lack of data available for north Indian population.

The study included 200 normal healthy individuals. Serum Iron \& Total iron Binding capacity (TIBC) levels of all subjects were estimated using kits supplied by Span diagnostics. DNA was extracted by Standard Phenol Chloroform method. Polymerase chain Reaction, Restriction Enzyme digestion was performed for identification of C282Y \& H63D mutations. Statistical analysis was performed.

No case of C282Y mutation Hetrozygosity or Homozygosity has been witnessed. One case of Homozygous mutation and 21 cases for Hetrozygous mutation for H63D have been witnessed.

H63D mutation is more common than C282Y mutation in North Indian population. H63D mutation normally results in only small rise in Iron store, which is not clinically significant. 
Indian Journal of Clinical Biochemistry, December 2007, Vol. 22 (Supl.)

P1.5

\section{Genetic Characterization of Mycobacterium Avium Subspecies Paratuberculosis Strain S5 and its Comparison with Map K10 Strain}

Jagdeep Singh Sohal', Neelam Sheoran', Swati Subodh', K Narayanasamy', Vani Brahmachari ${ }^{3}$, Shoorvir Singh ${ }^{2}$

Centre for Genomic Application', Delhi; Central Institute for Research on Goats², Mathura, D.B.R. Ambedkar Centre, Delhi ${ }^{3}$

Mycobacterium avium subspecies paratuberculosis (MAP) is the etiological agent of Johne's disease (JD or paratuberculosis); of ruminants characterized by chronic inflammation of intestine and weight loss. MAP strain 'S5' was isolated from an Indigenous goat breed of Northern India exhibiting advance clinical symptoms of JD. The MAP has been initially characterized as 'Bison type' and is most prevalent strain of North India infecting domestic and wild ruminants, including human population. This study was designed to conduct comparative genomic analysis of the architecture of the local MAP 'bison type' (S5) isolate with reference to IS elements, large sequence polymorphisms (LSPs) and short sequence repeats (SSRs).

Three main molecular markers were selected to characterize the MAP S5 strain, viz. Insertion elements (IS elements; IS900, IS1311, ISMAP02), SSRs (G repeat and GGT repeat) and LSPs; (LSPP2, LSPP4, LSPP12, $L_{S P} 15, L^{A} 20$ and LSPA4II.), based on genomic sequence amplification. The amplicons were characterized by sequencing. Bioinformatics tools were utilized for comparative analysis with MAP K10 sequences available. Southern hybridization was performed for each locus of IS900 and ISMAP02. In addition MAP strains from other field isolates were studied for SSR and IS1311 profiles.

Of the 17 IS900 and 6 ISMAPO2 loci present in MAP K 10, 15 and 5 loci could be amplified, respectively with $5^{\prime}$ end locus specific primers. All 7 IS1311 loci were amplified by $5^{\prime}$ end locus specific primers. In comparison with IS elements of MAP K10 only single nucleotide variations were observed.

With in the sequence of IS1311 sequences a TG dinucleotides gap was observed at $63^{\text {rd }}$ and $64^{\text {th }}$ position. This alteration was observed at 3 different loci; IS 1311 L2, IS1311 L5 and IS 1311 L6. For locus IS1311 L2 four Indian and one Portuguese isolate were studied where TG Gap was observed in MAP S5 strain .

In SSR profile Indian Map 'Bison type' isolate contained only 7 and 4 in place of 19 and 5 of Map K 10 of G Repeat and GGT repeats, respectively. The LSP profile of the two strains i. e. Map K10 and Indian MAP S5 isolate were same for all LSPs tested. Both the strains contained LSPP2, LSPP $4, L^{2} P^{P} 12$, LSPP 15 and LSPA20 and lack LSPA4 4 II.

Southern hybridization revealed that all 17 loci for IS900 were conserved and six copies of ISMAPO2 element have been observed was expected for MAP K10. Locus I of ISMAP02 showed positional polymorphism compared to Map K10 and appeared at higher size.

The MAP isolate 'Bison type', showed a C to T conversion at $223^{\text {rd }}$ position. 
Indian Journal of Clinical Biochemistry, December 2007, Vol. 22 (Supl.)

The consistent TG Gap characteristic of MAP S5 strain is a potential signature to distinguish MAP strains of Indian origin. Similarly the SSR profile for Map isolates of Indian origin from different hosts also suggests the characteristic feature of Indian Map isolates. Another observation of unexpected size band at MAPO2 (Locus 1) suggested the polymorphism at this locus.

P1.6

\title{
Effects of Total protein and Lipid on serum electrolytes measurements by Direct and Indirect lon Selective Electrode Measuring system
}

Monika Chadha

SPS Apollo Hospital, Ludhiana

lon selective electrodes (ISE) measure electrolytes viz., sodium, potassium and chloride by two different technologies, direct and indirect ISE. Direct ISE measures the active electrolyte in the water phase of the serum without any dilution of the serum samples. In Indirect ISE, electrolytes are measured in the volume of total serum and not the serum water phase, in the pre-diluted serum samples. The serum sample volume consists of liquid (which contains the dissolved electrolytes) and solids (e.g., proteins and lipids). Indirect ISE systems are serum. When the concentration of solids in serum are changed in samples, (e.g., lipemic or samples having very high or very low protein), electrolytes measurement may get affected which impact the clinical judgment.

A concurrent analysis of serum electrolytes was performed at our centre on 100 consecutive patients from January 2007 till July 2007. In this study, electrolytes were measured in serum samples with various levels of proteins and lipids by both Direct ISE method (Vitros 250 Dry Chemistry system) and by Indirect ISE method (Beckman CX 5) to understand the effect of protein and lipid on serum electrolytes measurement by both ISE methods. The parameters included in the study were Sodium, Potassium and Chloride.

We conclude that serum electrolytes are affected in a non-linear manner with the changes in serum protein or lipid concentration by indirect ISE systems because of electrolyte exclusion effect in such samples. In border-line clinical situations, direct ISE systems provide a more accurate measurement of electrolytes viz., sodium, potassium, chloride, anion gap and osmolality, which helps in better clinical decision making.

P1.7

\section{Application of Short Tandem Repeat Polymorphisms Present on Intron 6 of the LPL Gene for Individualiztion in Forensic Science}

\author{
V.R. Rathod, M.M. Bapat
}

The Institute of Science, Biochemistry Department, Mumbai.

The study includes the determination and distribution of alleles and genotype frequencies for short tandem repeat (STR) of Lipoprotein lipase (LPL) gene in the three major religious groups (Hindus, Muslims and Christians) in Mumbai. 
Indian Journal of Clinical Biochemistry, December 2007, Vol. 22 (Supl.)

The DNA was extracted from blood stains by using organic extraction method and was amplified by Polymerase chain reaction (PCR) using fluorescent based primer specific for intron 6 of the LPL gene. $A B I$ 3100 automated sequencer was used for the detection of the amplified products. Genotype results were analyzed using GENEMAPPER software.

No deviation from Hardy-Weinberg equilibrium was observed for allele and genotype distribution. Allele 9 was the most common and highly distributed allele in all the three religions. The most common homozygote genotype was $(9,9)$ and the most common heterozygote genotypes were $(10,9)$ and $(11,9)$. The homozygosity percentage was 29.7 and heterozygosity percentage was 70.3. The discrimination power 0.888 was observed.

LPL marker is highly polymorphic. 70\% heterozygosity of alleles and genotype distribution suggests that it can be used as an effective tool for human identification in Indian population. It is the first report of its kind for the population of Mumbai.

P1.8

\title{
Comparative analysis of the copy number and position of genomic landmarks of Mycobacterium tuberculosis clinical isolates.
}

\author{
Monalisa Ray', Swati Subodh', Monika Joon², K Narayanasamy \\ 1 The Centre for Genomic Application, New Delhi. \\ 2 B.R. Ambedkar Centre for Biomedical Research, University of Delhi, Delhi
}

Clinical isolates typing of mycobacterium has been an important component of epidemiological studies of tuberculosis and the insertion element like IS 6110 have been used as gold standard for identification. Considerable variations reported in the number and the size in the RFPL analysis of IS6 110 elements can mediate rearrangement and deletion in the genome. The recent report of monocyte activated promoter activity in the IS61 10 could contribute to position dependent-alteration in gene expression. In the background of this contention we undertook an analysis of the number and position of IS6110 in clinical isolates of M.tuberculosis. For generating a clinical isolate repository we have also analysed the specific Mycobacterium Interspersed Repetitive Unit VNTR and 16s r DNA.

For IS 61 10, we developed a multiplex PCR to identify its position with H37Rv sequence as reference. The same primers were used for PCR amplification of all clinical isolates; the amplicons were sequenced and compared with that of H37Rv for relative positioning. For the 12 different loci for MIRU in H37Rv, we used a multiplex PCR with one unlabeled and one fluorescence labeled primer in four sets of three MIRU VNTR loci. Sizing of the PCR fragments and assignment of the various MIRU-VNTR alleles were done using the GeneScan and customized Genotyper software packages. The amplification of 16s (r) DNA was done in a master mixture containing site specific primers and the amplicons were sequenced and phylogenetic analysis was done by nearest neighbor method.

Compared to the 16 bands pattern of H37Rv (positive control) bands are produced in H37Rv, the band pattern found in clinical isolates were variable. On aligning with H37Rv genome, some of 16 loci showed 
Indian Journal of Clinical Biochemistry, December 2007, Vol. 22 (Supl.)

deletion between the IS element and M.tb genome, some showed mismatch in genome part, some were properly aligned in the same position as H37Rv. In MIRU result, two (second and third multiplex) out of four multiplex were showing some different patterns. 16S(r) DNA PCR amplification gave same 875 bp amplicons.Sequence Identity Matrix(SIM) analysis showed 99\% similarity among the isolates.

Data obtained from IS6 110 concludes that though 16 IS elements are present in H37Rv, in clinical samples few are present at the same position \& others are present at different places of the genome.MIRU results infer that H37Rvand all clinical isolates differ in second \& third multiplex either in unit length of VNTR or in number of repeat units. As in 16S (r) DNA the SIM analysis shows almost $99 \%$ sequence similarity, thereby inferring that they are identical Mycobacteria/Strains.

P1.9

\section{Serum Alpha-2-Macroglobulin - A Tool for the Diagnosis of Ectopic Pregnancy}

Maya Roche, Saritha Kamath U, Cijo Paul, Mohit Sikka, Cinta Rose, Nalini K

Melaka Manipal Medical College, Manipal University, Manipal

An ectopic pregnancy (EP) occurs when implantation of the embryo occurs outside the uterus. If left untreated, the developing fetus will continue to grow, leading to life-threatening consequences for the mother. A major difficulty with the diagnosis of EP is that methods of detection are limited, and some, such as ultrasound, are not very reliable in the early days of gestation. The incidence of EP is increasing and has doubled in the last 20 years. It is now the second most common cause of maternal death in the first trimster of pregnancy. Currently, no effective serum test exists to distinguish an EP from a normal intrauterine pregnancy (NIUP). The present study attempts to assess the efficacy of serum alpha-2-macroglobulin $(\alpha 2 M)$ as a tool in the early diagnosis of EP.

Immunoreactive $\alpha 2 M$ concentrations were estimated by single radial immunodiffusion in serum sampels of normal nonpregnant women in the age group of 25-35 years, in women with NIUP and women with EP. NIUP and EP samples were selected based on positive $\beta$-hCG in serum and 4-7 weeks of pregnancy calculated from last menstrual period (LMP).

$\alpha 2 \mathrm{M}$ in the normal nonpregnant women was $398 \pm 28.98 \mathrm{mg} / \mathrm{dL}$, decreasing in normal intrauterine pregnancy (NIUP), $190.0 \pm 16.99 \mathrm{mg} / \mathrm{dL}$. In EP $\alpha 2 \mathrm{M}$ was significantly elevated, $1530+577 \mathrm{mg} / \mathrm{dl}$, $(p<0.001)$, when compared NIUP and non pregnant women $(p<0.001)$. As early as 4 weeks from the LMP when ultrasound findings are inconclusive, estimation of $\alpha 2 \mathrm{M}$ in serum can be used to differentiate a NIUP from EP. This will aid early diagnosis and treatment and prevent morbidity. 
$\mathrm{P} 1.10$

\title{
Predictive of Elevated Urinary Albumin Excretion in De Novo Development of Renal Function Impairment in the General Population
}

\author{
Uday Kumar, Anand Sharan
}

Dept. of Biochemistry, Indira Gandhi Institute of Medical Sciences, Patna, Bihar

The purpose of the study is to establish the important factor which determines the risk for developing renal function imparment. We studied the incidence of newly diagnosed impaired renal function, [Glomerular filtration rate (GFR) $<60 \mathrm{ml} / \mathrm{min}$ ] in the 2148 out door patients, with presence of albuminuria in 19992000. Subjects were put under survelliance for four years.

Subjects with known renal disease, GFR $<60 \mathrm{ml} / \mathrm{min}$, missing GFR values, or sediment abnormalities at the first screening were excluded from the present analysis $(\mathrm{N}=218)$. We examined whether albuminuria is associated with the de novo development of an impaired renal function. GFR was $90.3( \pm 16.3) \mathrm{ml} / \mathrm{min}$ at base line, and $11.6 \%$ of the subjects had an albuminuria of more than $30 \mathrm{mg} /$ day. After a followup of our years, 63 subjects $(4.2 \%)$ were found to have a GFR $<60 \mathrm{ml} / \mathrm{min}$. The subjects with newly diagnosed impaired GFR were older, had a higher blood pressure, serum cholesterol, plasma glucose, and urinary albumin excretion at the first examination, and had a lower GFR to start with than those with a GFR>60 at the second elevation. Subjects with de novo impaired GFR had a comparable body mass index (BMI) and smoked less frequently compared with subjects with GFR $>60$. In multivariate analysis, urinary albumin excretion was or independently predictive factor for the risk of developing an impared GFR $(P=0.001)$. Albuminuria is a strong predictor of diminished renal function de novo. The risk attributed to albuminuria is independent from cardiovascular risk factors. Albuminuria may, therefore, in the general population also prove to be a valuable risk marker for future renal function decline amenable for screening purposes.

P1.11

\section{Cartilage Oligomeric Matrix Protein (COMP), A Marker of Cartilage Destruction in Rheumatoid Arthritis}

\author{
A.N. Vinod', Nalini Ganesan', Rajasekar² \\ 1 Department of Biochemistry, ${ }^{2}$ Department of Rheumatology \\ Sri Ramachandra Medical College and Research Institute, Porur, Chennai.
}

Rheumatoid arthritis (RA) is an autoimmune disorder of unknown cause. The pathogenesis is characterized by chronic inflammation of the joint tissue leads to destruction of the joints followed by functional impairment. The emergence and use of molecular markers is one of the advances in the treatment of rheumatoid arthritis. With the development of molecular markers our therapeutic approach to rheumatoid arthritis and inflammatory disease in general has dramatically changed in the last few years. 
Indian Journal of Clinical Biochemistry, December 2007, Vol. 22 (Supl.)

Cartilage oligomeric matrix protein (COMP) is a tissue specific non-collagenous glycoprotein which binds with type II collagen fibers and stabilizes the collagen fiber network in the articular cartilage. An interesting feature of COMP is it release from cartilage into the blood during the erosion of the tissue in RA. Inflammatory synovial cartilage has been considered as a possible source of serum COMP since the molecule has been detected in the synovium in RA.

COMP level in serum should be useful for evaluating prognosis in patients with early rheumatoid arthritis and also as a marker for monitoring the therapy response in patients with RA. The present review article will provide an insight into the role of COMP as a potential molecular marker of cartilage destruction in the different aspects of disease process and its usefulness in early detection of rheumatoid arthritis. In our department we are planning to estimate a panel of markers of synovium, cartilage and bone in RA patients to elucidate their involvement in the pathogenesis of rheumatoid arthritis.

$\mathrm{P} 1.12$

\title{
DNA Based Diagnosis of Bacterial Meningitis
}

\author{
Manoi K. Patel ${ }^{1}$, Sunil Gupta ${ }^{2}$, Shashi Khare' ${ }^{2}$, Ashok Kumar ${ }^{1}$ \\ ${ }^{1}$ Institute of Genomics and Integrative Biology, Mall Road, Delhi. \\ ${ }^{2}$ National Institute of Communicable Diseases, Sham Nath Marg, Delhi.
}

Meningitis is a serious infection of the fluids in the spinal cord and the fluid surrounds the brain. It is usually caused by an infection with a virus or a bacterium. Virual meningitis is usually mild and it clears up within a week or two without treatment whereas bacterial meningitis is serious and cause brain damage and even death. Presently bacterial meningitis is diagnosed by CSF, X-ray, CT scan, blood test, biochemical test and PCR. The usual methods used in Indian hospitals are latex agglutination, Gram staining and biochemical test. Our objective is diagnosis of Neisseria meningitidis in CSF fluid by identifying virulent genes using rapid PCR.

Meningococcal disease is endemic in Delhi and other states of India. The clinical samples were tested for the diagnosis of the meningococcal meningitis including cerebrospinal fluid (CSF), blood and blood broth. Hospitals in Delhi and outside of Delhi send their samples of suspected individuals for diagnosis of the disease at NICD. During February 2007 to March 2007, 80 clinical samples were received for testing out of which 14 cases were found positive for the bacteria. Latex agglutination test and Grams staining was carried out with the CSF and from cultured bacteria in different media The samples were also screened by the different biochemical test like catalase, oxidase and antibiotic susceptibility test. The capsullar transferase virulent gene of N.meningitidis was identified and primer were designed and synthesized for direct identification of meningitis in CSF samples.

The genomic DNA was isolated by STE method from CSF samples of cultured bacteria in specific media. The PCR was carried out at desired temperature using genomic DNA and method was standardized. Efforts were made to identify N.meningitidis without isolating genomic DNA from CSF samples. In this method, CSF sample was directly taken for PCR with certain modifications in protocol to get quick results. The present method of diagnosis of meningitis is quick, accurate and based on virulent gene of neisseria meningitidis. 
$\mathrm{P} 1.13$

\title{
Detection of mutations and $5 \mathrm{~T}$ variant in the cystic fibrosis transmembrane conductance regulator (CFTR) gene in Indian patients with obstructive azoopsermia
}

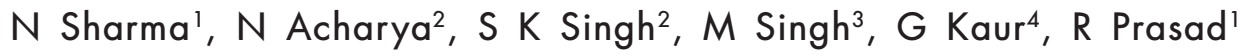 \\ ${ }^{1}$ Department of Biochemistry; ${ }^{2}$ Department of Urology; ${ }^{3}$ Department of Pediatrics, PGIMER, Chandigarh; \\ ${ }^{4}$ Department of Physiology, Government Medical College and Hospital, Chandigarh
}

Cystic Fibrosis (CF) is an autosomal recessive disorder caused by mutations in cystic fibrosis transmembrane conductance regulator (CFTR) gene. More than $95 \%$ of adult CF males are infertile. Although isolated azoospermia is a distinct clinical entity, but some males with this disorder may have an unusually mild form of CF. Till date, there is no report on the spectrum of CFTR mutations in Indian patients with genital form of CF. Therefore, the present study was conducted to investigate the spectrum of mutations in CFTR gene, their association with marker haplotypes and to establish genotype and phenotype correlation.

A prospective cohort study was conducted in infertile male subjects $(n=35)$ due to obstructive azoospermia. Female partners were also included. The diagnosis was made on physical examination, radiological findings and pathological evidence of normal spermatogenesis with nil sperm count. Sweat chloride was estimated by pilocarpine iontophoresis. The most common delta F508 and other known mutations were detected by either ARMS PCR or restriction digestion. All 27 exons and exon- intron boundaries of CFTR were screened by SSCP analysis.

Clinical data revealed bilateral $(n=25)$ or unilateral $(n=10)$ absence of vas deferens, absence of seminal vesicle $(n=10)$ and unilateral absence of kidney $(n=5)$. Mean sweat chloride value was $63.0 \mathrm{mEq} / \mathrm{L}$. Eight different mutations were identified in this study. Delta F508 was the most common mutation (31.4\%). Five novel mutations were characterized. Polymorphic $5 \mathrm{~T}$ allele was found in $27 \%$ of these subjects. All analyzed delta F508 chromosomes carried the same KM.19- GATT-TUB9-M470V-T854T haplotype. 4332-4333 Ins $G$ mutation in homozygous state was in one patient with pulmonary symptoms. In others, either heterozygous delta F508 or compound heterozygous genotypes with mild allele were identified.

We conclude that genital form of CF exists in India and the spectrum of mutations is heterogenous. 
$\mathrm{P} 1.14$

\title{
Standardization of PCR Based Diagnostic Test for Bovine Tuberculosis
}

\author{
Savita Kulkarni, P. R.Chaudhari, M.G.R. Rajan \\ Laboratory Nuclear Medicine Section, BARC, Parel, Mumbai.
}

Bovine tuberculosis is a debilitating disease of cattle. Its occurrence in a herd leads to major disruption of milk production and also in breeding programme. Apart from the economic losses, it is a source of probable transmission of the animal tuberculosis to humans. Diagnosis of the disease is currently based on a relatively insensitive and nonspecific Tuberculin skin test. The accepted gold standard test viz., invitro culture of pathogen which is time consuming ( 4 to 6 week) and is often negative from samples like blood and milk.

The study was undertaken to fulfill the need for sensitive and specific diagnostic test for bovine tuberculosis

A PCR test for detecting bovine tuberculosis was standardized using INS1 and INS2 primers amplifying $245 \mathrm{bp}$ segment of insertion sequence IS6110, having a sensitivity of $10 \mathrm{fg}$ of M.bovis DNA. DNA extraction procedures were standardized for milk and blood samples after spiking them with M.bovis cells ranging from 10 to 105 cells. To evaluate the usefulness of the test, blood and milk samples of cattle from two farms were screened.

Thirty blood and 20 milk samples were collected from Farm-I, and from Farm (II), 79 blood and 27 milk samples were tested.

From Farm-I 17 blood samples and 3 milk samples showed positive PCR results. From Farm-II 6 blood samples were clearly PCR positive and 16 of the blood samples showed equivocal results. None of the milk samples showed positive PCR results. This screening of blood samples was performed isolating PBMC from heparinized blood and then extracting DNA. When a modified DNA extraction protocol was used for extracting DNA directly from the heparinized blood samples from Farm (II), 13 out of 79 samples showed PCR positive results. Thus 7 blood samples which showed equivocal results with earlier protocol for DNA extraction, showed positive PCR results with modified protocol.

A sensitive PCR test for bovine tuberculosis has been standardised and the DNA extraction protocol was standardized using spiked samples. Further validation of both using clinically confirmed cases of bovine tuberculosis is under way. 
$\mathrm{P} 1.15$

\title{
Maximizing the Diagnostic and Therapeutic Impact of Serological Tests- case in Point Dengue
}

\section{R. Krishnaprasad}

DDRC-WELLSPRING Pathlab and Diagnostics, Ulloor Medical College PO, Thiruvananthapuram.

Serological detection of antibodies against infectious diseases has been shown to be an effective method in identifying infection. There are a number of commercial kits for detecting $\lg G$ and $\lg M$ antibodies. The accuracy and performance in routine settings of these kits vary due to differences in the reference materials used. This paper talks about ways to maximize diagnostic accuracy and cost effectiveness in serological testing with our experience with testing for Dengue

Prior to testing a discussion was had with the Physicians to determine what exactly they were expecting from the assay. Criteria were determined to stratify patients. Samples were obtained from patients with symptoms of Dengue and non symptomatic controls. Samples were analyzed by EIA and correlated with hematological findings and reported as positive/negative. The patients were followed up to assess the clinical outcome.

Setting up a pretest protocol increased the diagnostic and therapeutic efficacy of serological tests by allowing the treating physicians to focus on the small percentage of patients who are actually at risk. The establishment of a new cutoff value, taking into account the endemicity, allowed us to improve the specificity of the test.

The high cost of medical care is creating a trend to make more accurate, cost-effective clinical decisions. While laboratory tests aid in the assessment of disease probability, incorrectly ordered or improperly done tests merely add to the costs with no benefit. The usefulness of a laboratory test should also be based on the diagnostic and therapeutic impact and patient outcome as well. Clinicians to understand that tests should be selected and interpreted in a way that will allow them to estimate the probability of a cure.

P1.16

\section{Measurement of Urine and Plasma Oxalate with Reusable Strip of}

\author{
Nisha Sharma, Manikashi Sharma, V. Kumar, C.S. Pundir* \\ Biochemistry Research Laboratory, Deptt of Biochemistry \& Genetics, M.D. University, Rohtak.
}

Measurement of oxalate in urine and plasma is required in the diagnosis and medical management of urinary stone disease and various form of lipids malabsorption. The objective of the present study was to prepare a reusable strip of oxalate oxidase purified from Amaranthus leaves for measurement of oxalate in biological fluids.

A reusable strip of oxalate oxidase was prepared by immobilizing covalently partially purified Amaranthus leaf oxalate oxidase on to alkalys amine glass beads affixed on a plastic strip. A method for discrete Indian Journal of Clinical Biochemistry, December 2007 
Indian Journal of Clinical Biochemistry, December 2007, Vol. 22 (Supl.)

analysis of oxalate in urine and plasma was developed employing this enzyme strip. The method is based on measurement of $\mathrm{H}_{2} \mathrm{O}_{2}$ generated from urinary/plasma oxalate by strip bound oxalate oxidase using a colour reaction consisting of 4-aminophenazone, phenol and horseradish peroxidase as chomogenic system. The strip was reused after washing it with reaction buffer $(0.05 \mathrm{M}$ sodium citrate buffer $\mathrm{pH} 3.5)$ and stored in the same buffer at $4^{\circ} \mathrm{C}$ when not in use.

The immobilized enzyme gave a conjugation yield of $48 \mathrm{mg} / \mathrm{g}$ support with $87 \%$ retention of initial activity of free enzyme. The strip showed maximum activity at $\mathrm{pH} 3.5$ when incubated at $40^{\circ} \mathrm{C}$ for $15 \mathrm{~min}$. The minimum detection limit of the method was $0.1 \mathrm{mM}$. The recovery of added oxalate was $96.5 \%$ in plasma and $98 \%$ in urine. Within and between assay coefficient of variation were $<6 \%$ and $<5 \%$ respectively in plasma and urine. The oxalate value in $24 \mathrm{~h}$ urine and plasma in apparently healthy adult males and females as determined by the present method, were in the range of 11.5 to $27.5 \mathrm{mg} / \mathrm{L}$ with a mean of $20.80 \mathrm{mg} / \mathrm{L}$ and 2.5 to $3.8 \mu \mathrm{mol} / \mathrm{L}$, with a mean of $2.94 \mu \mathrm{mol} / \mathrm{L}$ respectively. The strip bound enzyme did not show any noticeable change in its activity up to 40 days during its regular use (200 times).

A simple, sensitive specific method for discrete analysis of oxalate in urine and plasma was developed using reusable strip of Amaranthus leaf oxalate oxidase.

$\mathrm{P} 1.17$

\section{$A B C D I$ Gene Polymorphism in X-linked Adrenoleukodystrophy in Indian Population}

Neeraj Kumar ${ }^{1}$, K.K. Taneja ${ }^{2}$, Veena Kalra ${ }^{3}$, Madulika Kabra $^{4}$, S. Aneja ${ }^{5}$, Madhur Behari ${ }^{6}$, S.K. Bansal'

${ }^{1}$ Dept. of Biochemistry, V.P. Chest Insititute, University of Delhi, Delhi.

2 Institute of Eenomics and Integrative Biology, Delhi

${ }^{3}$ Dept. of Pediatrics, ${ }^{4}$ Dept. of Genetics

${ }^{5}$ Dept. of Pediatrics, Lady Hardinge Medical College, New Delhi

${ }^{6}$ Dept. of Neurology, All India Institute of Medical Sciences, New Delhi

Adrenoleukodystrophy is a complex genetic recesive, peroxisomal severe neurodegenerative, progressive disorder which affects the adrenal gland and white matter of the brain. It is caused by mutations in the $A B C D 1$ (ATP-Binding cassette, sub-family D) gene which encodes a peroxisomal $A B C$ half-transporter (ALDP) involved in the import of very long-chain fatty acids (VLCFA) into the peroxisome. The aim of the study was to identify and establish genetic association of $A B C D l$ gene polymorphism with various phenotypes of adrenoleukodystrophy.

A total number of 13 adrenoleukodystrophy patients and 70 non-Adrenoleukodystrophy, ethinically unrelated matched healthy subjects, as controls, were enrolled. The patients were recruited on the basis of MRI or VLCFA analysis. Blood was collected, genomic DNA isolated, PCR for $A B C D 1$ gene and sequencing performed using $A B I 3100$ automated sequencer.

Five single nucleotide polymorphisms (one 5', three intronic and one exonic) were identified in controls 
Indian Journal of Clinical Biochemistry, December 2007, Vol. 22 (Supl.)

and adrenoleukodystrophy patients. We identified 5' UTR polymorphism C/T at position -59 (frequency $16.67 \%, T-6 / 36$ ) and exonic polymorphism G/A at position 1548 (frequency 18.6\%, A-13/70) in Indian population. We also identified intronic polymorphism $G / A$ at position- 105 with a frequency of $5.4 \%(2 / 37)$ and $C / T$ at position -16 with a frequency of $5.4 \%(2 / 37)$ and another novel unique polymorphism G/T at position -51. SNP results were analyzed for association with various phenotypes of adrenoleukodystrophy. However, statistically significant association of any of the five polymorphisms was not observed.

Our results shows that $A B C D$ lgene polymorphisms alone might not be an important risk factor for Adrenoleukodystrophy patients. This disorder is showing a complex phenotypic expression and it is not possible to explain this variation only on the basis of single gene. Apparently more than one gene seems to be involved in this complex disease whose protein products may interact with the ALDP.

$\mathrm{P} 1.18$

\title{
Complete screening of the CFTR gene in Indian Cystic Fibrosis patients
}

\author{
Tester F. Ashavaid, Rani Raghavan
}

Research Laboratories, P.D. Hinduja National Hospital \& Medical Research Centre, Mumbai.

A $3 \mathrm{bp}$ deletion in CFTR exon 10, resulting in the loss of phenylalanine at position 508 in the gene product $(\Delta \mathrm{F} 508)$ has been reported to have a frequency of approximately $19-56 \%$ in Indian patients. None of the other CF mutations have been reported in our population. So, in order to detect other common molecular defects, we will secreen the entire 27 exons of the CF gene including the promoter and intronic regions.

Single Stranded Confromation Polymorphism (SSCP) of Polymerase Chain Reaction OPCR)-amplified gene segments is being used for CF gene screening. By varying temperature, gel composition, ionic strength and additives, we will optimize the sensitivity of SSCP for the CFTR gene screening. The presence of heteroduplexes may also be detected on most gels and in some cases contribute to the ability to distinguish certain mutations.

This method permits rapid detection of any sequence change in a given DNA segement and is being used successfully to analyze the CFTR gene. A study of CFTR exons 1, 4, 6b, 8, 9, 10, 11, 13b, 14a, 15, 19 and 21 so far has detected 3 mutations including $\Delta \mathrm{F} 508$ mutation in exons $6 \mathrm{~b}, 10$ and $14 \mathrm{~b}$. These nucleotide variations have been identified by comparing altered electrophoretic behavior together with positive control DNA samples previously characterized by others as well as negative healthy control DNA samples.

The strategy presented here will consititute a valuable tool for the development of CF tgeting for suspected CF individuals. The data obtained from this study will consequently be important for the development of adequate strategies for the molecular diagnosis of CF in India. 
Indian Journal of Clinical Biochemistry, December 2007, Vol. 22 (Supl.)

$\mathrm{P} 1.19$

\title{
Cytogenetic and Yq microdeletion analysis in couples experiencing recurrent IVF failure
}

\author{
Rima Dada1, Rakesh Kumar, Venkatesh Sundararajan, R K Sharma² \\ ' Laboratory for Molecular Reproduction and Genetics, Deptt. of Anatomy,All India institute of Medical \\ Sciences, ${ }^{2}$ ART centre, Army Research and Referral hospital, New Delhi.
}

Background Infertility is the lack of pregnancy after one year of regular unprotected sexual intercourse. About 15-20 couples harbour genetic abnormalities. These Chromosomal abnormalities in infertile couples results in spermatogenic arrest, premature ovarian failure, implantation failure and consequently failure of InVitro fertilization (IVF).

The aim of the study was to determine genetic basis for recurrent ART/IVF failure.

Fifty four infertile couples with IVF failure having poor blastocyst development and implantation were analyzed cytogenetically and for molecular analysis ofAZF loci in the men.

Two females with recurrent IVF failure showed partial deletion of Xq, three had deletion in the Xp22.3-24, and the other female had 10 cell line showing deletion of pericenteromeric region of long arm of chromosome number 1. Four men had chromosomal abnormality. Out of this two had a translocation in the D-D group chromosomes and two had balanced reciprocal translocations on autosomes. Of these couples microdeletion analysis of 50 cytogenetically normal infertile men, only two cases showed deletion; one with AZFc loci and the other case had deletion of AZFb loci. The couples where female partner had deletion of long arm of $X$ chromosome(Xq-) resulted in repeated failure of blastocyt development, in 4 IVF cycles. The case with AZFb microdeletion had maturation arrest and case with AZFc deletion had hypospermatogenesis. In these cases sperms could be retrieved from the testis and to be used for IVF or Intracytoplasmic sperm injection. (ICSI). In cases with sex chromosomal and autosomal aberrations there is probability of poor embryo development and consequently poor implantation, which may be a result of high segregation abnormalities and may negatively affect the outcome of assisted reproductive techniques.

ART is a very expensive technique and recurrent ART/IVF failure results in severe financial stress coupled with emotional stress, thus all couples opting for ART must undergo genetic analysis. 